\title{
Tecnura
}

\section{Efecto del grado de capacidad de disipación de energía sísmica seleccionado en las cantidades de obra de muros de concreto reforzado}

\section{Effect of the selected seismic energy dissipation capacity on the materials quantity for reinforced concrete walls}

\author{
José Miguel Benjumea Royero ${ }^{1}$, Fredy Saúl Sotelo Monroy²,Camilo Eduardo Celis Melo ${ }^{3}$ \\ Gustavo Chio $\mathrm{Cho}^{4}$
}

Fecha de recepción: 1 de abril de 2016

Fecha de aceptación: 10 de septiembre de 2016

Cómo citar: Benjumea, J.M.; Sotelo, F.S.; Celis, C.E. y Chio, G. (2016). Efecto del grado de capacidad de disipación de energía sísmica seleccionado en las cantidades de obra de muros de concreto reforzado. Revista Tecnura, 20(50), 15-28. doi: 10.14483/udistrital.jour.tecnura.2016.4.a01

\section{RESUMEN}

Contexto: Para el diseño de muros estructurales de concreto reforzado, la normativa sismoresistente en Colombia permite, en función de la amenaza sísmica del sitio, optar por uno de tres posibles grados de capacidad de disipación de energía (mínima, moderada y especial). Sin embargo, la práctica empleada es la de adoptar el menor grado acorde a la amenaza sísmica.

Método: En este trabajo se realizó un estudio analítico del efecto del grado de capacidad de disipación de energía seleccionado durante el diseño de muros estructurales de concreto reforzado, en las cantidades de obra de la estructura y en la capacidad de desplazamiento. El estudio se centró en zonas de amenaza sísmica baja, ya que permite explorar y comparar los tres grados de disipación de energía. En el estudio se incluyeron otras variables como la altura total del muro, el área tributaria y la cuantía mínima de refuerzo adoptada para el alma del muro.
Resultados: la cantidad total de acero requerida para los muros con capacidad moderada y especial corresponde, en promedio, a un $77 \%$ y $89 \%$, respectivamente, de la cantidad requerida para los muros con capacidad mínima.

Conclusiones: es posible lograr reducciones en el peso total de acero requerido al emplear grados de capacidad de disipación moderada y especial comparado con el uso de capacidad de disipación mínima. Además, con esta estrategia se lograr un incremento significativo en la ductilidad del muro.

Palabras Clave: Amenaza Sísmica Baja, Capacidad de desplazamiento, Capacidad de Disipación de Energía, Cantidades de obra, Elementos de borde, Muros de concreto.

\section{ABSTRACT}

Context: Regarding their design of reinforced concrete structural walls, the Colombian seismic design building code allows the engineer to select one of

1 Ingeniero civil, magíster en Ingeniería Civil, profesor asistente de la Universidad Industrial de Santander. Bucaramanga, Colombia. Contacto: josbenro@uis.edu.co

2 Ingeniero civil, magíster en Ingeniería Civil, director de Diseño Estructural Asgard Ingeniería y Construcción. Bucaramanga, Colombia. Contacto: sote_sote@hotmail.com

3 Ingeniero civil, gerente Asgard Ingeniería y Construcción. Bucaramanga, Colombia. Contacto: camiloedo.celis@gmail.com

4 Ingeniero civil, doctor ingeniero en Caminos, Canales y Puertos. Profesor titular de la Universidad Industrial de Santander. Bucaramanga, Colombia. Contacto: gchioch@uis.edu.co 
the three seismic energy dissipation capacity (ordinary, moderate, and special) depending on the seismic hazard of the site. Despite this, it is a common practice to choose the minor requirement for the site because it is thought that selecting a higher requirement will lead to larger structural materials amounts and, therefore, cost increments.

Method: In this work, an analytical study was performed in order to determine the effect of the selected energy dissipation capacity on the quantity of materials and ductility displacement capacity of R/C walls. The study was done for a region with low seismic hazard, mainly because this permitted to explore and compare the use of the three seismic energy dissipations capacities. The effect of different parameters such as the wall total height and thickness, the tributary loaded area, and the minimum volumetric steel ratio were studied.

Results: The total amount of steel required for the walls with moderate and special energy dissipation capacity corresponds, on average, to $77 \%$ and $89 \%$, respectively, of the quantity required for walls with minimum capacity.

Conclusions: it is possible to achieve reductions in the total steel required weight when adopting either moderated or special seismic energy dissipation instead of the minimum capacity. Additionally, a significant increment in the seismic ductility displacements capacity of the wall was obtained.

Keywords: Boundary elements, Displacement ductility capacity, Energy Dissipation Capacity, Low Seismic Regions, Quantity of materials, RC Walls

\section{INTRODUCCIÓN}

Con el fin de reducir las pérdidas humanas y materiales ante un evento sísmico, el reglamento colombiano de construcción sismorresistente, en adelante NSR-10, establece requisitos mínimos de diseño para elementos estructurales en función del sistema estructural, los materiales usados, el desempeño sísmico esperado de la edificación y el nivel de amenaza sísmica. Los dos últimos parámetros están estrechamente relacionados ya que en función de la amenaza sísmica del sitio, el proyectista dispone de diferentes grados de capacidad de disipación de energía sísmica en el rango inelástico (tabla 1). Sin embargo, a la hora de diseñar, la práctica común consiste en seleccionar los requisitos mínimos de disipación exigidos para un nivel de amenaza sísmica dado. Por ejemplo, en ciudades con amenaza sísmica baja, como Barranquilla, generalmente se emplean requisitos de disipación de energía mínimos (DMI).

Una posible alternativa para el diseño sería seleccionar un mayor grado de capacidad de disipación de energía sísmica que el mínimo requerido acorde al nivel de amenaza sísmica del sitio. Este enfoque podría parecer inviable, desde el punto de vista económico, ya que el incremento del grado de capacidad de disipación de energía se hace, principalmente, mediante mayores restricciones en el empleo de los materiales (por ejemplo, con

Tabla 1. Opciones de grados de capacidad de disipación de energía en función de la amenaza sísmica establecidos en NSR-10

\begin{tabular}{lccc}
\hline \multirow{2}{*}{ Capacidad de disipación de energía } & \multicolumn{3}{c}{ Zona de amenaza sísmica } \\
\cline { 2 - 4 } & BAJA & MEDIA & ALTA \\
\hline Mínima (DMI) & $\checkmark$ & $\checkmark$ & \\
\hline Moderada (DMO) & $\checkmark$ & $\checkmark$ & $\checkmark$ \\
\hline Especial (DES) & $\checkmark$ & & \\
\hline
\end{tabular}

Fuente: AIS (2012). 
un mejor detallamiento del refuerzo transversal en estructuras de concreto). En teoría, esto incrementaría las cantidades de obra de la estructura; sin embargo, al seleccionar un grado de capacidad de disipación de energía mayor que el mínimo especificado, las fuerzas sísmicas de diseño obtenidas de un análisis lineal y elástico tendrán una mayor reducción, ya que el coeficiente de capacidad de disipación (R), especificado en la tabla A.3-1 de NSR-10, aumenta. Por tanto, el refuerzo longitudinal requerido podría disminuir y generar un equilibrio en el consumo total de materiales.

En Colombia, debido al creciente uso de muros delgados de concreto reforzado, las investigaciones se han enfocado en estudios analíticos (Blandón y Bonett, 2013; Naranjo, Marulanda y Madera, 2015) y experimentales (Arteta y Moehle, 2015; Blandón, Rave y Bonett, 2015) del desempeño sísmico de este tipo de muros. Además, se ha estudiado el comportamiento de edificaciones con muros de concreto reforzados de baja altura (Carrillo, Sánchez-Cruz y Viviescas, 2014), y se ha explorado el uso del diseño basado en desplazamientos para esta tipología estructural (Urrego y Bonett, 2010). Sin embargo, hasta la fecha no se han reportado análisis con el objetivo de determinar los efectos de seleccionar un grado de capacidad diferente al mínimo especificado para la zona sísmica.

Teniendo en cuenta el planteamiento previo y el hecho de que el número de edificaciones que emplean muros estructurales en concreto reforzado como sistema de resistencia sísmica viene creciendo paulatinamente en el país (Blandón y Bonett, 2013), se plantea la pregunta de investigación: ¿Cuál es el efecto en las cantidades de obra de la estructura en edificaciones con muros estructurales al seleccionar un grado de capacidad de disipación de energía diferente al mínimo establecido para un nivel de amenaza sísmica dado? La presente investigación está centrada en zonas de amenaza sísmica baja, ya que permite explorar y comparar todas las capacidades de disipación de energía disponibles en el código para el sistema estructural; sin embargo, el estudio también se podría replicar para zonas de amenaza sísmica intermedia. En este artículo, tras una descripción de la metodología empleada, se presentan los principales resultados, en los que se enfatiza en las cantidades de obra de estructura y en la capacidad de ductilidad de desplazamiento del muro. Este último parámetro, aunque no es el objetivo principal de la investigación, fue incluido con el fin de contar con una medida adicional que permitiera evaluar la decisión de seleccionar un grado de capacidad de disipación de energía diferente al mínimo exigido por NSR-10. Por último, se exponen las principales conclusiones del estudio.

\section{METODOLOGÍA}

De acuerdo con Wood (2005), para el caso de edificaciones cuyo sistema de resistencia de fuerzas sísmicas son los muros estructurales, el análisis puede ser desarrollado mediante un modelo simplificado como el presentado en la figura 1. Para dicho muro, la respuesta estructural ante cargas laterales dependerá de la altura total $\left(h_{w}\right)$ y de entrepiso $\left(h_{\text {piso }}\right)$, las dimensiones en planta $\left(\mathrm{L}_{w} \mathrm{y} t_{w}\right)$, la rigidez a flexión $\left(\mathrm{E}_{c} I\right)$, donde $\mathrm{E}_{c}$ es el módulo de elasticidad del concreto e I es el momento de inercia respecto al eje mayor, y el índice de muros de la edificación $(\beta)$, definido como la relación entre el área en planta del piso y la sumatoria del área de la sección transversal de todos los muros, o, para el caso del modelo conceptual, la relación entre el área tributaria y el área de la sección transversal del muro. 


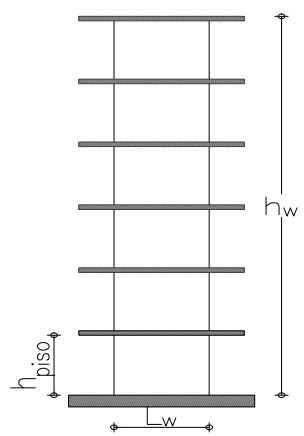

a) Vista Lateral

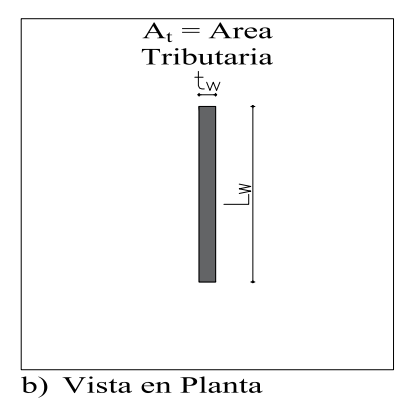

Figura 1. Modelo conceptual empleado en el análisis.

Fuente: elaboración propia.

Con el fin de representar la práctica en el medio colombiano, en este estudio fueron seleccionados tres muros prototipos con una altura de entrepiso igual a 2,55 m, y alturas totales de 15,3 m, 30,6 m y $45,9 \mathrm{~m}$, que corresponden a 6, 12 y 18 pisos respectivamente. Para cada caso, el espesor del muro $\left(t_{w}\right)$ fue modificado entre $0,15 \mathrm{~m}$ y $0,30 \mathrm{~m}$, con incrementos de $0,05 \mathrm{~m}$. La longitud del muro $\left(L_{w}\right)$ fue determinada de modo que los requisitos de deriva y resistencia establecidos en NSR-10 sean verificados. En relación al área tributaria, se emplearon dos valores fijos $\left(24\right.$ y $45 \mathrm{~m}^{2}$ ) con el fin de capturar el comportamiento para densidades de muros altas y bajas, respectivamente. El estudio se limitó a muros fundidos en sitio, excluyendo muros prefabricados en donde las conexiones entre los elementos requieren de consideraciones especiales.

En general, la metodología empleada inicia con el cálculo de las cargas gravitacionales y laterales. A continuación se ejecuta el análisis estructural para determinar la longitud del muro que satisfaga el requisito de deriva permitido (deriva de entrepiso máximo igual al $1 \%$ de la altura de piso). Luego se realiza el diseño del muro siguiendo los requisitos de los capítulos C.14 y C.21 de la NSR-10, aplicando los requerimientos de ductilidad en función del grado de disipación de energía seleccionado. Por último, se determinan las cantidades de obra de la estructura y la capacidad de ductilidad de desplazamiento para cada muro. Estos pasos se describen a continuación.

\section{Acciones}

Las solicitaciones consideradas se determinaron de acuerdo con los requisitos del título B establecidos en NSR-10, tomando como cargas muertas (D) una carga sobreimpuesta de $3,0 \mathrm{kN} / \mathrm{m}^{2}$, el peso propio de la placa maciza de espesor asumido igual a $0,15 \mathrm{~m}$, y el peso propio de los muros. Además, se incluyeron la carga viva (L) de magnitud 1,8 kN/ $\mathrm{m}^{2}$ y las fuerzas sísmicas de diseño (Fs) distribuidas en altura, siguiendo el procedimiento establecido en el capítulo A.4 de la NSR-10. Para la definición del espectro de aceleración, presentado en la figura 2, se asumió que la construcción se hace en una ciudad con coeficientes $A_{a}=0,1$ y $A_{v}=0,1$ y suelo tipo D. Cabe resaltar que en el presente estudio no se incluyó la interacción entre el suelo y la estructura. Adicionalmente, debido a que el estudio se centra en los efectos sísmicos, no se incluyeron otras acciones como las cargas de viento. Las combinaciones de carga usadas para el diseño de los muros se presentan en la tabla 2. Las fuerzas sísmicas reducidas de diseño (E) se obtuvieron mediante la división de las fuerzas sísmicas (Fs) por el coeficiente de disipación de energía $(R)$ para cada grado de disipación seleccionado. Estos últimos se establecen en la tabla A.3-1 del código NSR-10 y corresponden a 2,5, 4 y 5 para los grados de disipación DMI, DMO y DES, respectivamente.

Tabla 2. Combinaciones de resistencia última empleadas

\begin{tabular}{lccc}
\hline \multirow{2}{*}{$\begin{array}{c}\text { Combinación según } \\
\text { NSR-10 }\end{array}$} & \multicolumn{3}{c}{ Factor de mayoración } \\
\cline { 2 - 4 } & $\mathbf{D}$ & $\mathbf{L}$ & $\mathbf{E}$ \\
\hline B.2.4-1 & 1.4 & & \\
\hline B.2.4-2 & 1.2 & 1.6 & \\
\hline B.2.4-5 & 1.2 & 1.0 & 1.0 \\
\hline B.2.4-6 & 0.9 & & 1.0 \\
\hline
\end{tabular}

Fuente: AIS (2012). 


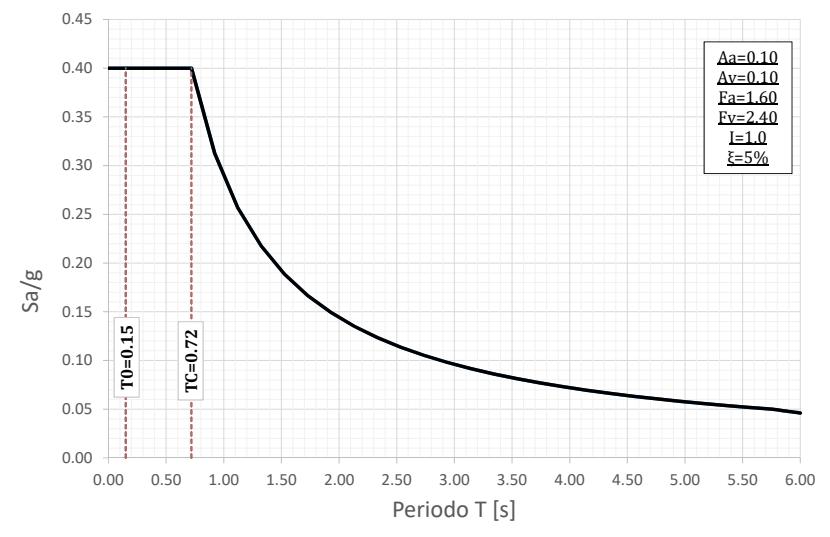

Figura 2. Espectros de aceleración sin reducir empleado

Fuente: elaboración propia.

\section{Modelo numérico y materiales empleados}

El análisis estructural se realizó mediante modelamiento numérico en el software SAP2000-v14.2.4. Los muros fueron analizados usando elementos tipo Shell (Computers and Structures Inc., 2009) y nodos articulados en la base. La selección de este tipo de condición de contorno obedece a que el modelo numérico fue discretizado generando múltiples nodos al nivel de cimentación, lo cual,

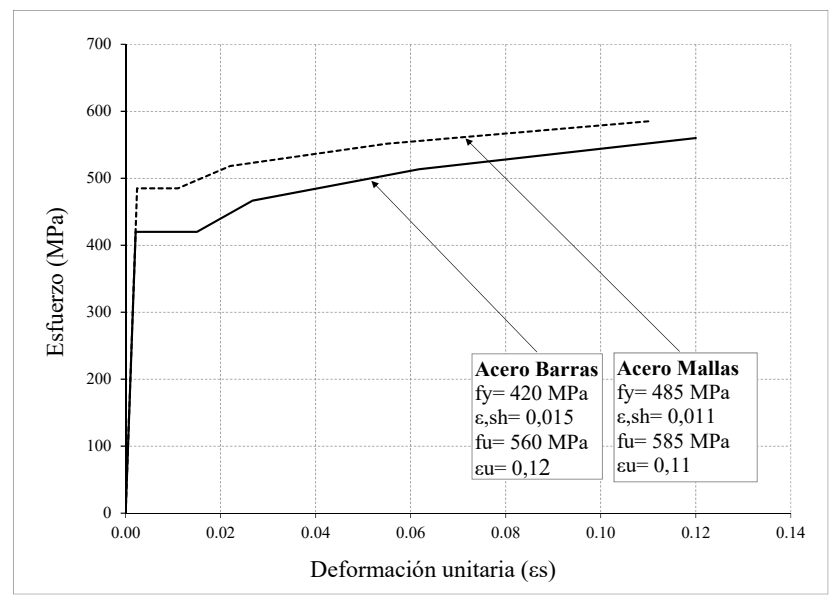

con el tipo de apoyo seleccionado, se logra una restricción de la rotación en dicho nivel. Durante la fase de diseño de los muros, se ejecutó un análisis lineal y elástico, de modo que las fuerzas sísmicas se pudieran posteriormente reducir mediante el coeficiente de disipación, R, como se explicó en la sección anterior. Sin embargo, con el fin de calcular la capacidad de desplazamiento de los muros, fue necesario realizar un análisis seccional teniendo en cuenta las relaciones constitutivas de los materiales. Para esto, se asumió un concreto con resistencia a la compresión $\left(f_{c}^{\prime}\right)$ igual a 28 $\mathrm{MPa}$, módulo de elasticidad $\left(E_{c}\right)$ de $21.000 \mathrm{MPa}$, coeficiente de Poisson (v) igual a 0,25 , y peso específico $(\gamma)$ de $24 \mathrm{kN} / \mathrm{m}^{3}$. El refuerzo consiste en acero no presforzado con módulo de elasticidad $\left(E_{s}\right)$ igual a $200.000 \mathrm{MPa}$. Para las barras de acero se usó un esfuerzo de fluencia $\left(f_{y}\right)$ igual a $420 \mathrm{MPa}$. En el caso del acero de mallas electrosoldadas se empleó un esfuerzo de fluencia igual a $485 \mathrm{MPa}$. Las relaciones esfuerzo-deformación del concreto no confinado y de los aceros se presentan en la figura 3. En estas, los términos $\varepsilon_{s h^{\prime}} \varepsilon_{u}$ y $f_{u}$ corresponden a la deformación unitaria en el punto de inicio de endurecimiento por deformación, deformación unitaria última, y esfuerzo último del acero, respectivamente.

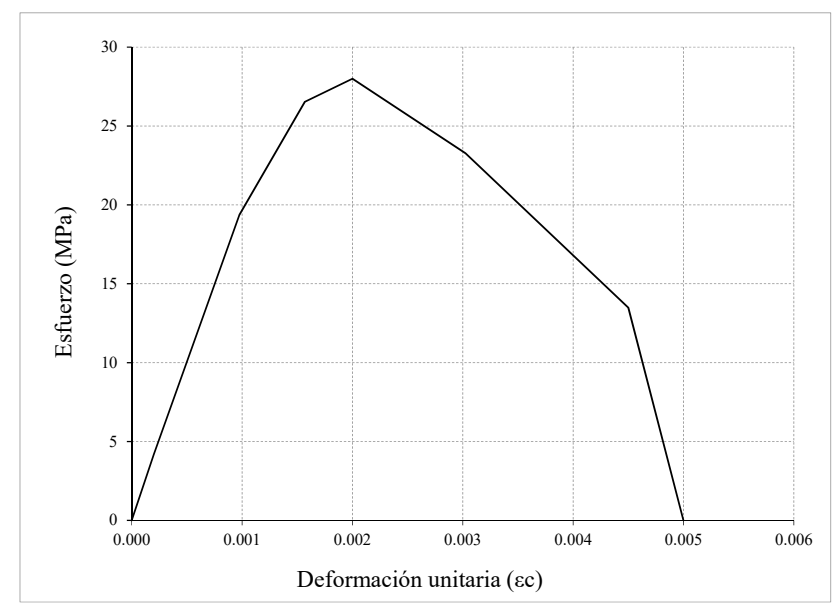

Figura 3. Relaciones esfuerzo deformación empleadas para el concreto y acero

Fuente: elaboración propia. 


\section{Diseño estructural}

Todos los muros fueron diseñados por cortante y flexocompresión de modo que cumplieran los requisitos de resistencia y ductilidad en función del grado de disipación de energía seleccionado. Para los muros con disipación mínima (DMI), los principales requisitos en relación a las cuantías longitudinales $\left(\rho_{\rho}\right)$ y transversales $\left(\rho_{t}\right)$ están gobernados por el capítulo C.14 de la NSR-10 y se resumen en la figura 4. En estos muros, cuando la cuantía longitudinal requerida era mayor o igual al $1 \%$, algo que ocurre en los primeros pisos cuando se emplean muros delgados, el refuerzo longitudinal fue confinado por estribos cerrados y ganchos suplementarios adicionales, separados de manera intermitente entre las barras longitudinales.

Por otra parte, en los muros con disipación moderada y especial, los requisitos en relación al refuerzo longitudinal y transversal están gobernados por las prescripciones del capítulo C.21 de la NSR-10. Los principales cambios en relación a los requisitos de muros con capacidad mínima son el uso de cuantías de acero longitudinal y transversal que dependen de la demanda a cortante $\left(V_{u}\right)$ (figura 5a), y la implementación de elementos de borde. Ya que en zonas como la del estudio en donde las demandas sísmicas son bajas, el uso de cuantías de refuerzo mayores o iguales a 0,25 \% podría ser excesivo para reducir la fisuras por tensión diagonal (Carrillo, González y Rubiano, 2014), el diseño de los muros se realizó para dos escenarios. En el primero, se permitió el uso de cuantías reducidas de refuerzo en el alma en función de la demanda a cortante. En el segundo, se empleó, como mínimo, una cuantía de refuerzo igual al 0,25\% (véase notas en figura 5a). Con el fin de determinar el número de pisos en donde se requieren los elementos de borde, en este estudio se seleccionó el enfoque de esfuerzos permisibles en la fibra extrema a compresión (figura 5b). El detallado del acero de confinamiento empleado en los elementos de bordes se presenta en la figura 6, donde:
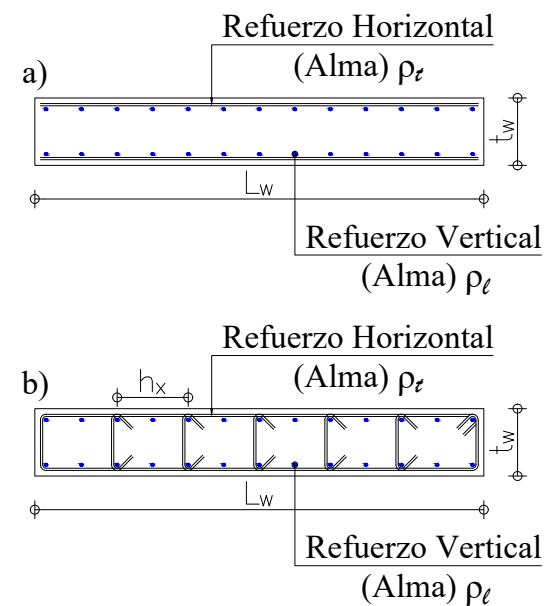

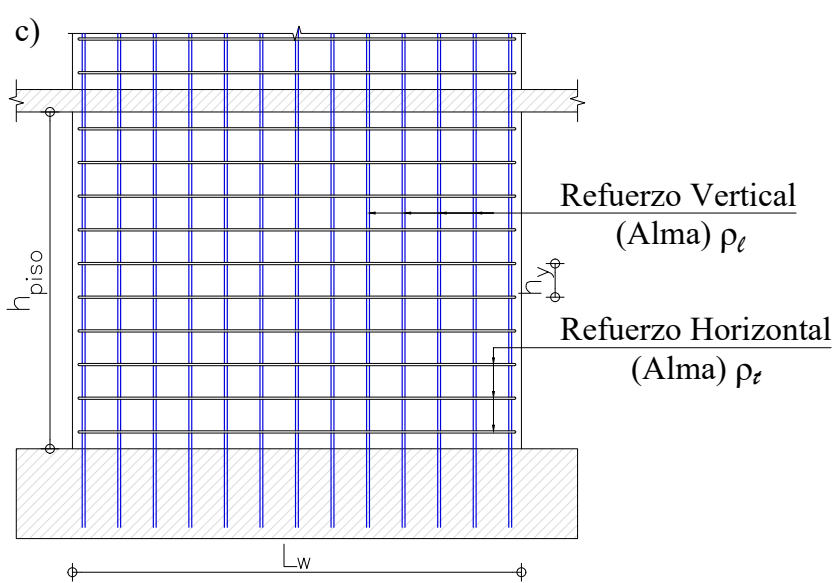

Notas

1. $\rho_{\ell}>0,0012$ (Sección C.14.3.2 NSR-10).

2. $\rho_{t}>0,0020$ (Sección C.14.3.2 NSR-10).

3. $h_{x} y h_{y}$ menor al minimo entre $3 t_{w}$ y $0,45 \mathrm{~m}$ (C.14.3.4).

Figura 4. Refuerzo típico en muros rectangulares con capacidad de disipación DMl: (a) sección típica, (b) sección con $\rho_{\mathrm{L}} \geq 1 \%$, (c) alzado y notas

Fuente: elaboración propia. 
- $A_{s h}=$ área total de refuerzo transversal colocado dentro del espaciamiento $s$ y perpendicular a la dirección $b_{c}$.

- $L_{b e}=$ longitud del elemento de borde.

- $S$ = separación del refuerzo transversal del elemento de borde.

- $h_{x}=$ espaciamiento máximo horizontal entre ganchos suplementarios o ramas de estribos cerrados de confinamiento.
- $b_{c}=$ dimensión transversal del núcleo del elemento medida entre los bordes extremos del refuerzo transversal con área $A_{s h}$.

- $\quad C=$ distancia medida desde la fibra extrema en compresión al eje neutro.

- $d_{b l}=$ diámetro de la barra longitudinal del elemento de borde.

- $d_{b e}=$ diámetro del estribo del elemento de borde.
Notas:

1. $\rho_{\ell}>0,0025$ (Sección C.21.9.2.1 NSR-10).

2. $\rho_{t}>0,0025$ (Sección C.21.9.2.1 NSR-10).

3. $\rho_{\ell}$ y $\rho_{t}$, se pueden reducir a lo prescrito en Sección

C.14.3 si: $V_{u}<0,083 \times A_{c v} \times \sqrt{f^{\prime}}$

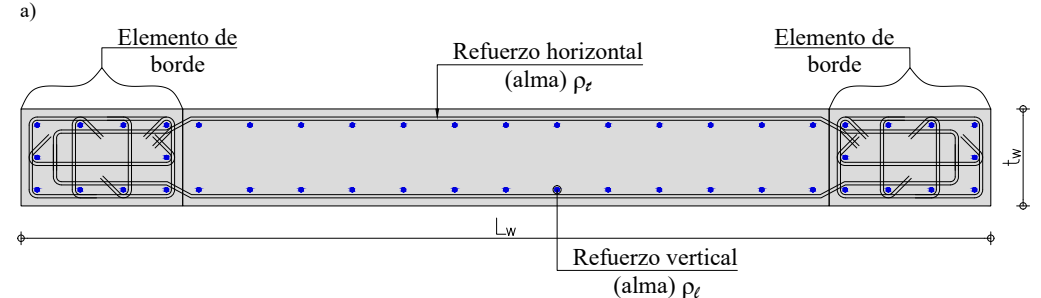

(alma) $\rho_{\ell}$

Vu : Fuerza cortante en la sección

Acv : Area bruta de la sección de concreto limitada por el espesor del alma y la longitud de la seccion en la direccion de la fuerza cortante considerada

b)

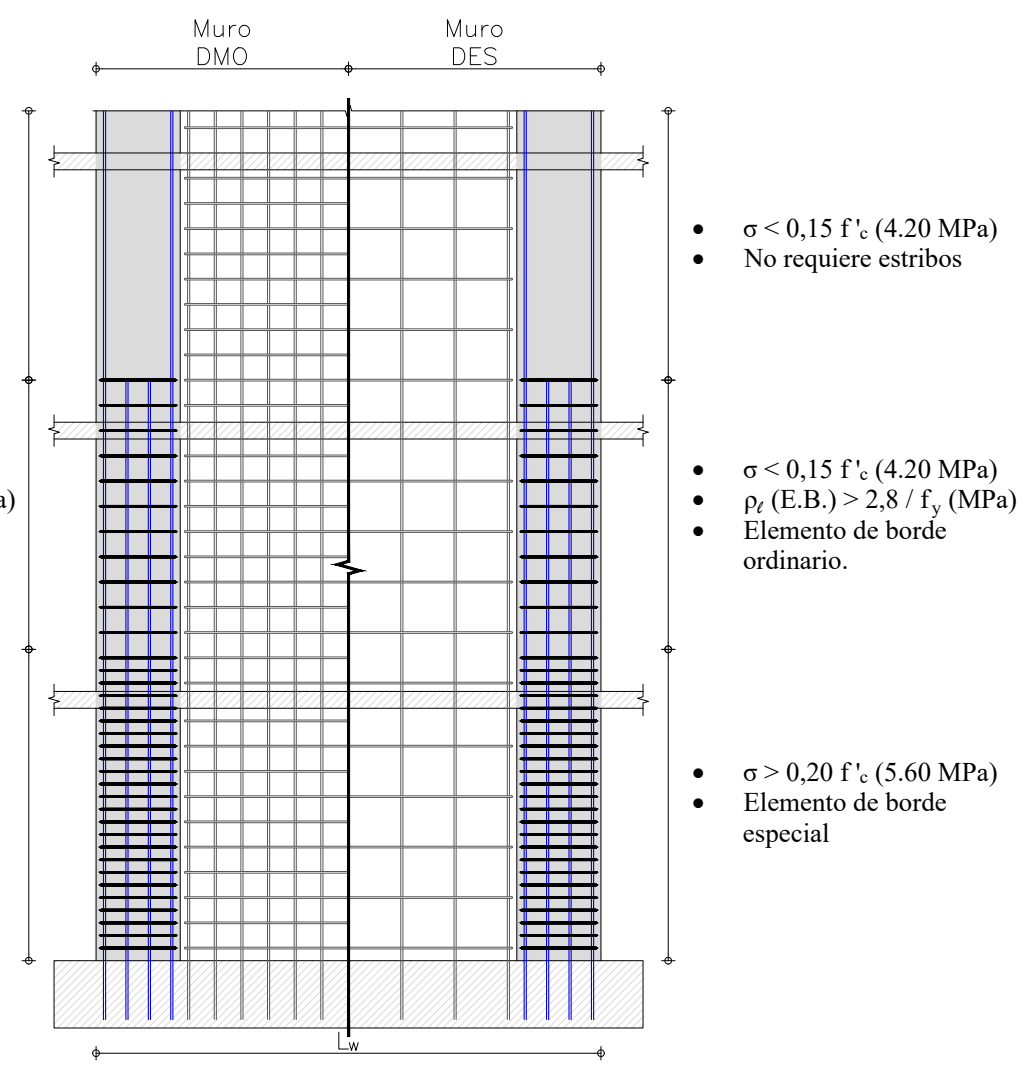

Figura 5. Requerimiento de refuerzo longitudinal y transversal para muros DMO y DES: (a) planta, (b) alzado

Fuente: elaboración propia. 
El refuerzo vertical requerido en los elementos de borde fue distribuido de modo que se cumplieran los requisitos de separación vertical y horizontal del refuerzo transversal. Además, como mínimo, se usó una cuantía longitudinal del $1 \%$ del área del elemento de borde, que corresponde a la cuantía mínima establecida por el código NSR-10 para columnas de concreto reforzado. Por último, con el fin de no generar cambios en el despiece de los elementos de borde en cada piso, algo que resultaría poco práctico a la hora de construir el muro, cuando fuese necesario, se optó por cambiar la sección y refuerzo del elemento de borde cada dos pisos.

Cabe resaltar que aunque el código NSR-10 permite usar dos capas de refuerzo a partir de espesores mayores a 0,25 $\mathrm{m}$ (en muros con grados DMI) o cuando la fuerza cortante mayorada sea superior a $0,17 A_{c v} \sqrt{f^{\prime} c}$ (en muros con grados DMO y DES), en el presente trabajo se empleó refuerzo en dos capas en todos los muros estudiados. Adicionalmente, cuando no se emplean elementos de borde y el refuerzo vertical se conforma con mallas, se optó por colocar dos barras de acero de diámetro 3/8" amarradas por ganchos del mismo diámetro separados cada $15 \mathrm{~cm}$.

\section{Cálculo de la ductilidad de desplazamiento}

Aunque el principal objetivo del presente trabajo es determinar la variación en las cantidades de obra de muros en función del grado de disipación de energía seleccionado, resulta interesante, además, comparar la capacidad de ductilidad de desplazamiento $\left(\mu_{\Delta}\right)$ del muro para cada grado de disipación. De esta manera se cuenta con un parámetro adicional que permitirá evaluar la decisión de seleccionar un grado de capacidad de disipación de energía diferente al mínimo exigido por la normativa.

La capacidad de ductilidad de desplazamiento se determina como la relación entre el desplazamiento último $\left(\Delta_{\mathrm{u}}\right)$ y el desplazamiento en fluencia $\left(\Delta_{\mathrm{y}}\right)$ del muro. Estos desplazamientos fueron a)

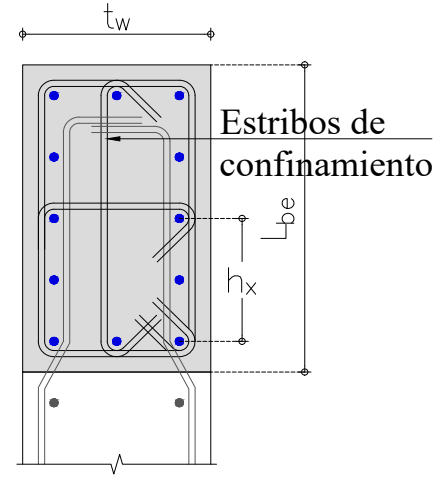

$$
\frac{A_{s h}}{S \times b_{c}} \geq \frac{0,09 f^{\prime}}{f_{y t}}
$$

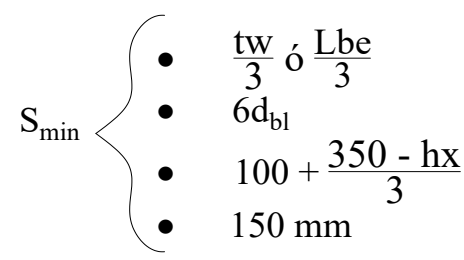

b)

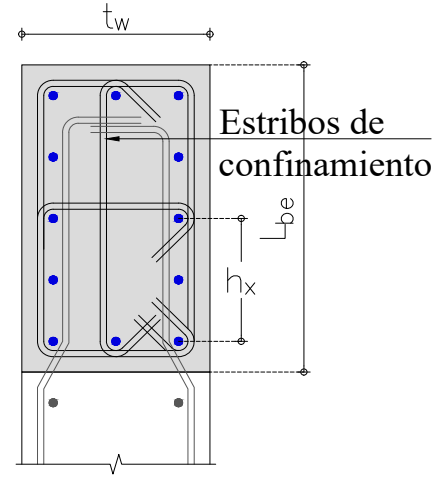

$$
\frac{A_{s h}}{S \times b_{c}} \geq \frac{0,06 f^{\prime}{ }_{c}}{f_{y t}}
$$

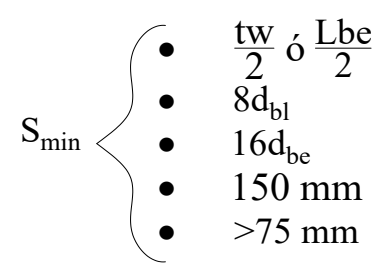

c)

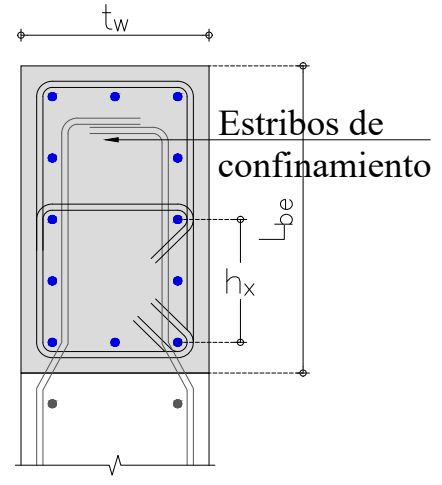

Notas:

1. $\mathrm{L}_{\mathrm{be}} \geq \max \left(\mathrm{c}-0,1 \mathrm{~L}_{\mathrm{w}} ; \frac{\mathrm{c}}{2}\right)$

2. $\mathrm{h}_{\mathrm{x}} \leq 350 \mathrm{~mm}$

Figura 6. Requerimiento de elementos de borde: (a) especial, (b) moderado y (c) ordinario

Fuente: elaboración propia. 
calculados mediante las ecuaciones (1) y (2), respectivamente. Debido a la esbeltez de los muros analizados y a que se buscaba una aproximación en los cálculos, solo se incluyeron los efectos de deformación por flexión. Los valores de la curvatura en el punto de fluencia efectivo $\left(\varnothing_{Y}\right)$ y punto último $\left(\varnothing_{u}\right)$ fueron obtenidos a partir de la idealización elastoplástica del diagrama momento-curvatura, realizado mediante el balance de áreas bajo las curvas (figura 7). Este diagrama fue obtenido para la carga axial esperada empleando la combinación D+0,25 L recomendada en (ASCE 41, 2013). La longitud equivalente de rótula plástica $\left(L_{p}\right)$ se tomó igual a $0,5 L_{w}$. Este valor es sugerido en Wallace (2007) para efectos prácticos, y, además, es usado implícitamente en la sección C.21.9.6.2 del código NSR-10 durante el cálculo de la mayor profundidad del eje neutro congruente con el desplazamiento de diseño.

$$
\begin{gathered}
\Delta_{u}=\Delta_{y}+L_{p}\left(\emptyset_{u}-\emptyset_{Y}\right)\left(h_{w}-\frac{L_{p}}{2}\right) \\
\Delta_{y}=\emptyset_{Y} \frac{h_{w}{ }^{2}}{3}
\end{gathered}
$$

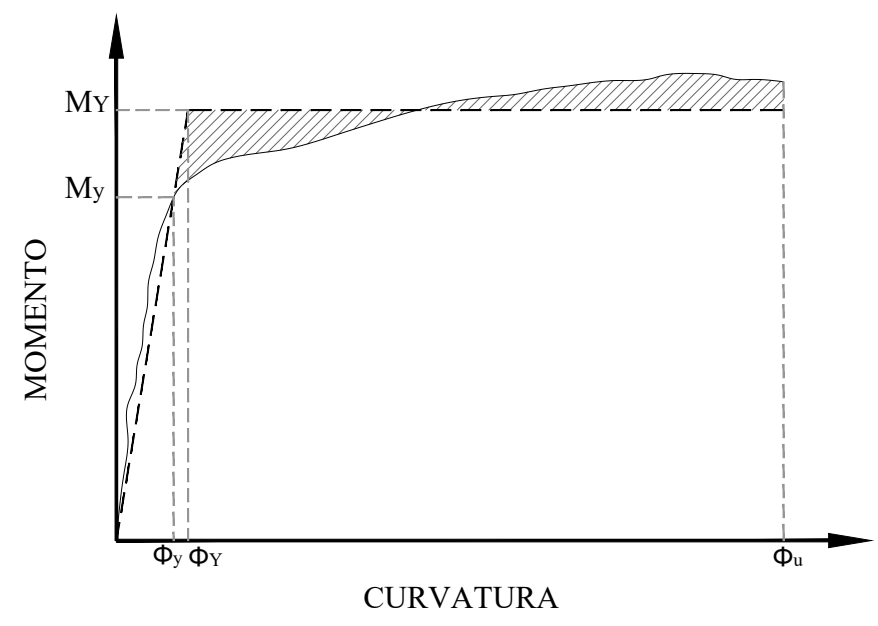

Figura 7. Idealización del diagrama momento curvatura empleado

Fuente: elaboración propia.
Las propiedades del concreto confinado se calcularon mediante el modelo propuesto por Mander, Priestley y Park (1994); sin embargo, en lugar de calcular el coeficiente de confinamiento efectivo $\left(k_{e}\right)$ con las ecuaciones propuestas por los autores, se usaron los valores recomendados por Abo-Shadi, Saiidi y Sanders (2000) presentados en la figura 8. Este coeficiente representa la fracción del área encerrada por la línea media de los estribos que está siendo confinada efectivamente.

\section{Casos analizados}

En la tabla 3 se presentan las características geométricas y el índice de muros para cada uno de los muros analizados. Además, se incluye el índice de carga axial (ALI) para el muro del primer piso, calculado mediante la ecuación (3). En la tabla también se incluye la relación $M /\left(V L_{w}\right)$ obtenida para las fuerzas sísmicas. El valor promedio de $M /\left(V L_{w}\right)$ es 5,3, lo cual refleja un comportamiento y una potencial falla de los muros gobernado por flexión (Hidalgo, Ledezma y Jordán, 2002).

$$
\text { A.L.I. }=\frac{P_{u}}{f_{c}\left(L_{w} t_{w}\right)}
$$

a) Muro DMI $\rho_{\ell}<1 \%$

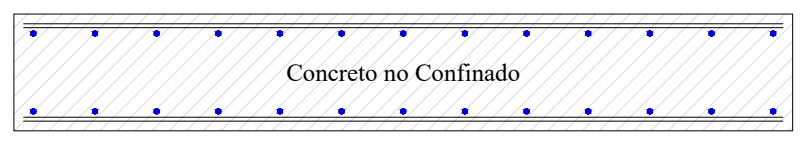

b) $\quad$ Muro DMI $\rho_{\ell}>1 \%$

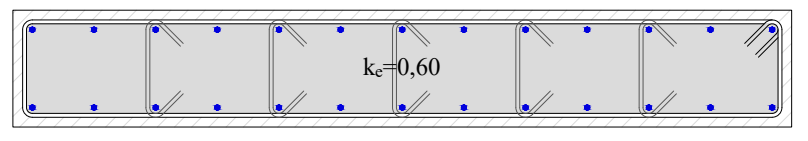

c) Muro DMO y DES

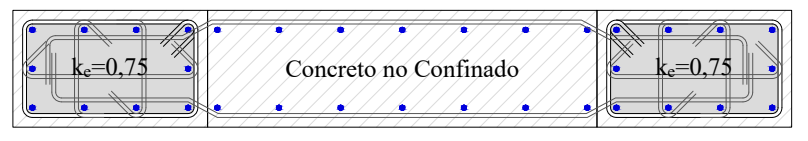

Figura 8. Factor de confinamiento efectivo empleado para el alma y los elementos de borde

Fuente: elaboración propia. 
Tabla 3. Propiedades de muros analizados

\begin{tabular}{|c|c|c|c|c|c|c|c|c|c|c|}
\hline Muro & $N p$ & $h_{w}(m)$ & $t_{w}(m)$ & $L_{w}(m)$ & $A_{t}\left(m^{2}\right)$ & $\frac{h_{w}}{L_{w}}$ & $\frac{L_{w}}{t_{w}}$ & $\beta$ & $\frac{M}{\overline{V L}}$ & A.L.I. \\
\hline 1 & \multirow{5}{*}{6} & \multirow{5}{*}{15.3} & 0,15 & 2,3 & 24 & 6,7 & 6,8 & $1,4 \%$ & 4,9 & 0,15 \\
\hline 2 & & & 0,20 & 2,0 & 24 & 7,7 & 7,7 & $1,7 \%$ & 5,5 & 0,14 \\
\hline 3 & & & 0,20 & 2,5 & 45 & 6,1 & 12,5 & $1,0 \%$ & 4,4 & 0,20 \\
\hline 4 & & & 0,25 & 1,9 & 24 & 8,1 & 8,1 & $2,0 \%$ & 5,8 & 0,12 \\
\hline 5 & & & 0,30 & 1,8 & 24 & 8,5 & 8,5 & $2,3 \%$ & 6,1 & 0,09 \\
\hline 6 & \multirow{5}{*}{12} & \multirow{5}{*}{30,6} & 0,15 & 4,5 & 24 & 6,8 & 6,8 & $2,8 \%$ & 4,8 & 0,18 \\
\hline 7 & & & 0,20 & 4,2 & 24 & 7,3 & 7,3 & $3,5 \%$ & 5,1 & 0,12 \\
\hline 8 & & & 0,25 & 3,9 & 24 & 7,9 & 7,9 & $4,1 \%$ & 5,5 & 0,13 \\
\hline 9 & & & 0,25 & 4,7 & 45 & 6,5 & 18,8 & $2,6 \%$ & 4,6 & 0,19 \\
\hline 10 & & & 0,30 & 3,7 & 24 & 8,3 & 8,3 & $4,6 \%$ & 5,8 & 0,12 \\
\hline 11 & \multirow{5}{*}{18} & \multirow{5}{*}{45,9} & 0,15 & 6,6 & 24 & 7,0 & 7,0 & $4,1 \%$ & 4,9 & 0,19 \\
\hline 12 & & & 0,20 & 6,1 & 24 & 7,5 & 7,6 & $5,1 \%$ & 5,3 & 0,13 \\
\hline 13 & & & 0,25 & 5,7 & 24 & 8,1 & 8,1 & $5,9 \%$ & 5,7 & 0,15 \\
\hline 14 & & & 0,30 & 5,5 & 24 & 8,4 & 8,4 & $6,9 \%$ & 5,9 & 0,13 \\
\hline 15 & & & 0,30 & 6,4 & 45 & 7,2 & 21,3 & $4,3 \%$ & 5,1 & 0,19 \\
\hline
\end{tabular}

Fuente: elaboración propia.

\section{RESULTADOS}

\section{Cantidades de acero de refuerzo}

Ya que las dimensiones de cada muro analizado no varían en función del grado de disipación de energía sísmica seleccionado, las cantidades de concreto requeridas no fueron analizadas. Por tanto, en esta sección se analizan los resultados del acero requerido para cada muro, los cuales son presentados en la figura 9. Con el fin de observar el efecto de los requisitos de ductilidad en los muros con capacidad moderada y especial, los cuales se centran en el incremento del confinamiento en los elementos de bordes, y la afectación del refuerzo transversal y longitudinal en el alma del muro debido a la variación del coeficiente de disipación de energía, el peso de acero fue desglosado en refuerzo vertical y horizontal requerido en el alma (L-Alma y T-Alma) y en los elementos de borde (L-EB y T-EB). La razón de que los muros con disipación de energía mínima presenten cantidades en refuerzo asociadas con elementos de borde en la figura 9 obedece a que, para este cálculo, las barras y los ganchos de diámetro 3/8" mencionados en la sección "Diseño estructural" fueron incluidos en este ítem.
En relación a la distribución del refuerzo requerido para los muros en función del grado de disipación de energía seleccionado, se encontró que en los muros con capacidad DMI, el refuerzo transversal y longitudinal en el alma corresponde a un promedio del $28 \%$ y $68 \%$, respectivamente, del total. En los muros con capacidad DMO esos porcentajes cambian al 31 $\%$ y $24 \%$, respectivamente, mientras que el refuerzo transversal y longitudinal en los elementos de borde corresponde en promedio a un $21 \%$ y $24 \%$ del total. Por último, en los muros con capacidad DES el refuerzo transversal y longitudinal en el alma representan un $27 \%$ y $21 \%$, respectivamente, del acero requerido. En estos muros, el refuerzo transversal y longitudinal en los elementos de borde es, en promedio, un $30 \%$ y $22 \%$ del refuerzo total.

Al comparar la cantidad total de acero requerida para los muros DMI y DMO, se encontró que en promedio, los segundos requieren un $77 \%$ del total de acero demandado por los muros con capacidad mínima. Esta reducción se logra por el menor acero requerido en el alma debido a la reducción en la demanda a flexocompresión y cortante, resultado directo del incremento en el coeficiente de capacidad de disipación de energía. La comparación de los muros DMI y DES reveló que el acero 
total requerido en los segundos es, en promedio, un $89 \%$ del total solicitado en los muros con capacidad mínima. La explicación a este resultado es similar a la presentada para los muros con capacidad moderada; sin embargo, en este caso hay una menor reducción ya que en los muros con capacidad especial se requirió una mayor cantidad de acero, debido a las mayores restricciones en el confinamiento en los elementos de borde. Por ejemplo, al comparar las cantidades de los muros con grado de capacidad de disipación de energía DMO y DES, se encontró que el refuerzo requerido en el alma del muro y el refuerzo vertical de los elementos de borde eran similares en ambos casos; sin embargo, los muros DMO requirieron, en promedio, de un $63 \%$ del acero transversal provisto en los elementos de borde de los muros DES. Este valor está dentro de lo estipulado en el comentario C.21.3.5 del código NSR-10, en el cual se expresa que los requisitos de confinamiento para columnas DMO fueron establecidos de modo tal que se tenga una cantidad de confinamiento equivalente al $60 \%$ de lo que se exige en columnas DES. De los casos analizados también se observó que el acero requerido en los muros DES sobrepasa o tiende a sobrepasar el requerido en los muros DMI cuando

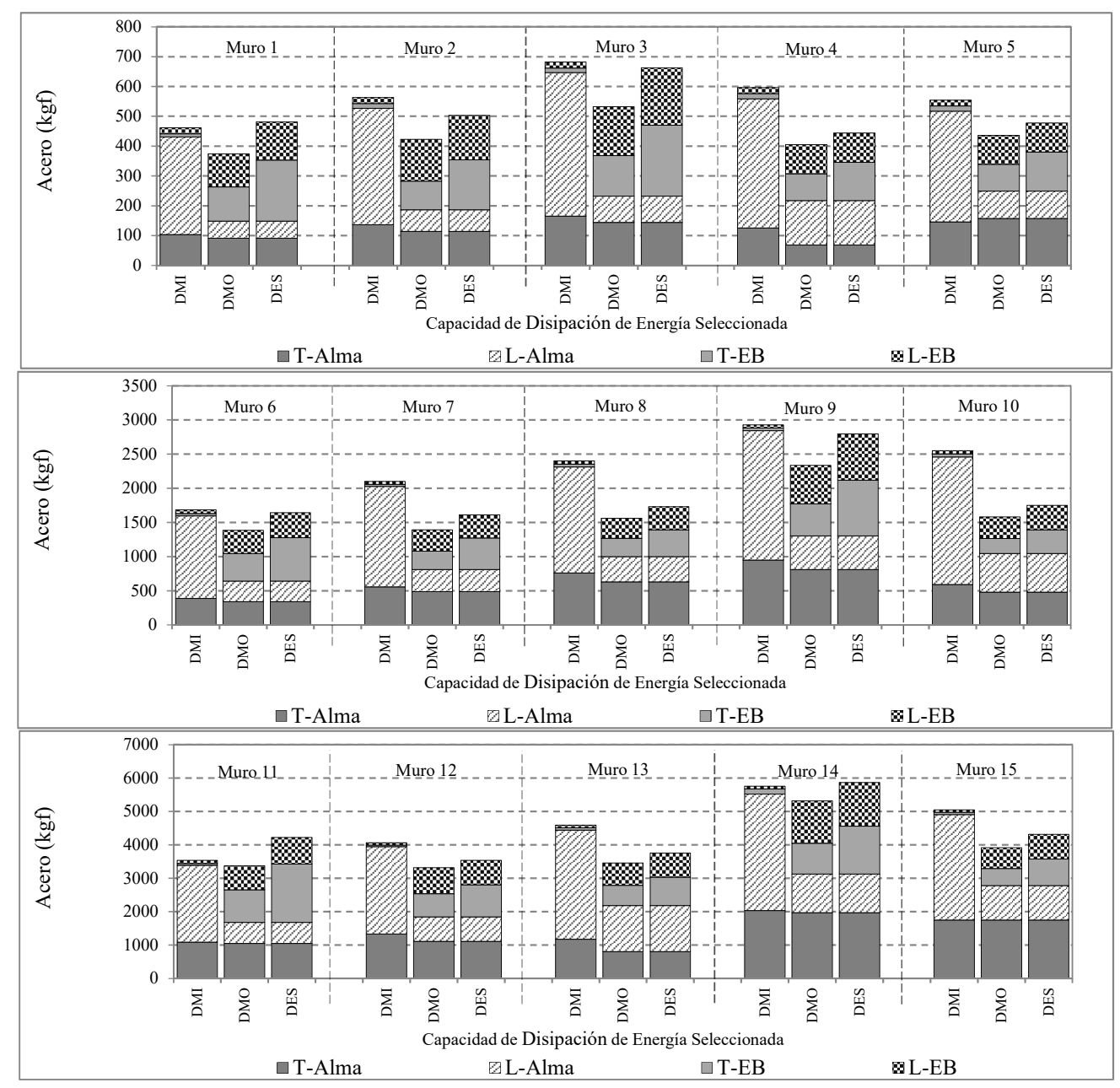

Figura 9. Peso de acero en función del grado de disipación de energía seleccionado: (arriba) 6 pisos, (centro) 12 pisos, (abajo) 18 pisos

Fuente: elaboración propia. 
se usan muros delgados $\left(t_{w}<0,15 \mathrm{~m}\right)$ y cuando el índice de muros es bajo (menor a $2 \%$ ).

Los resultados anteriores fueron obtenidos usando la reducción permitida en la cuantía longitudinal y transversal del refuerzo en el alma de los muros con capacidad moderada y especial, presentada en la nota 2 de la figura 5(a). Si se opta por usar una cuantía mínima igual al 0,25\% en ambas direcciones, el refuerzo total requerido en los muros se incrementa, siendo este efecto más notorio en los muros DMO y DES. Para este caso, las cantidades promedio de acero requeridas son iguales al $94 \%$ y al $106 \%$, respectivamente, de la requerida para los muros con capacidad DMI (tabla 4).

\section{Capacidad de ductilidad de desplazamiento}

En la tabla 5 se presenta la comparación de los resultados relacionados con el análisis descrito en la sección "Cálculo de la ductilidad de desplazamiento". Para los muros diseñados con grado de disipación DMI se observó que la capacidad de ductilidad de desplazamiento promedio es 3,2. Por otra parte, en los muros DMO y DES se obtuvieron valores promedios iguales a 7,4 y 7,5 respectivamente, demostrando así una mayor ductilidad global de la sección debido al efecto de los estribos de confinamiento en los elementos de borde. Lo anterior se confirma mediante la comparación del factor de confinamiento (K) obtenido para cada caso. Dicho factor representa la relación entre la resistencia a la compresión del concreto confinado $\left(f^{\prime}{ }_{c c}\right)$ y el concreto no confinado $\left(f^{\prime}{ }_{c}\right)$. Por ejemplo, para los casos (b) de la figura 8 , los factores $\mathrm{K}$ oscilaron entre 1,05 y 1,15, mientras que en los muros DMO y DES los valores promedio de $\mathrm{K}$ fueron 1,45 y 1,60 , respectivamente.

Si se confrontan los valores de desplazamiento en el punto de fluencia efectivo se observa que para los tres grados de disipación de energía los resultados son similares. Sin embargo, existe una diferencia importante en el desplazamiento último de los muros. Por ejemplo, en los muros DMO la capacidad de desplazamiento último es, en promedio, 2,5 veces mayor al obtenido en los muros
Tabla 4. Peso total de acero en kilos obtenido al emplear cuantías mínimas del 0,25\%

\begin{tabular}{cccc}
\hline Muro & $\mathbf{W}_{\text {DMI }}$ & $\begin{array}{c}\mathbf{W}_{\text {DMO }} \\
\left(\mathbf{W}_{\text {DMO }} / \mathbf{W}_{\text {DMI }}\right)\end{array}$ & $\begin{array}{c}\mathbf{W}_{\text {DES }} \\
\left(\mathbf{W}_{\text {DEs }} / \mathbf{W}_{\text {DMI }}\right)\end{array}$ \\
\hline 1 & 464 & $454(0,98)$ & $559(1,23)$ \\
\hline 2 & 578 & $524(0,91)$ & $604(1,15)$ \\
\hline 3 & 721 & $652(0,90)$ & $782(1,08)$ \\
\hline 4 & 616 & $537(0,87)$ & $577(1,07)$ \\
\hline 5 & 567 & $507(0,89)$ & $549(1,08)$ \\
\hline 6 & 1844 & $1572(0,85)$ & $1967(1,07)$ \\
\hline 7 & 2131 & $1799(0,84)$ & $2018(0,95)$ \\
\hline 8 & 2546 & $2163(0,85)$ & $2330(0,92)$ \\
\hline 9 & 3121 & $3128(1,00)$ & $3589(1,15)$ \\
\hline 10 & 2699 & $2213(0,82)$ & $2382(0,88)$ \\
\hline 11 & 3750 & $4153(1,11)$ & $5003(1,33)$ \\
\hline 12 & 4141 & $4235(1,02)$ & $4460(1,08)$ \\
\hline 13 & 4960 & $4808(0,97)$ & $5107(1,03)$ \\
\hline 14 & 5393 & $5178(0,96)$ & $5584(1,04)$ \\
\hline 15 & 6143 & $6730(1,10)$ & $7277(1,18)$ \\
\hline
\end{tabular}

Fuente: elaboración propia.

DMI, mientras que en los muros DES el factor aumenta a 2,6. Al comparar los valores del momento de fluencia efectivo $\left(M_{y}\right)$, que es concomitante con el valor de la curvatura $\left(\varnothing_{Y}\right)$ (figura 7 ), se observa que la resistencia nominal de los muros con capacidad mínima es, en promedio, 1,4 veces mayor a la resistencia de los muros con capacidad moderada y especial. Este es un resultado esperado ya que los muros DMI se diseñaron con un menor valor de coeficiente de disipación de energía y, por tanto, para resistir mayores efectos internos debido a las cargas laterales sísmicas.

Finalmente, si se decide emplear una cuantía mínima longitudinal y transversal en el alma del muro igual a $0,25 \%$, se encuentra que la capacidad de ductilidad de desplazamiento promedio obtenida para los muros DMO y DES es 6,51 y 7,12, respectivamente. La reducción en la capacidad de ductilidad de desplazamiento comparada con los datos presentados en el párrafo anterior obedece a la reducción en la curvatura última de la sección como resultado del incremento del refuerzo y, por tanto, en la reducción del desplazamiento último. Este resultado concuerda con lo 
Efecto del grado de capacidad de disipación de energía sísmica seleccionado en las cantidades de obra de muros de concreto reforzado Benjumea, J.M.; Sotelo, F.S.; Cells, C.E. y Chio, G.

Tabla 5. Comparación de la capacidad de desplazamiento y resistencia en función del grado de disipación de energía seleccionado

\begin{tabular}{|c|c|c|c|c|c|c|c|c|c|c|c|c|}
\hline \multirow[b]{2}{*}{ Muro } & \multicolumn{4}{|c|}{ Capacidad DMI } & \multicolumn{4}{|c|}{ Capacidad DMO } & \multicolumn{4}{|c|}{ Capacidad DES } \\
\hline & $\begin{array}{c}\Delta_{y} \\
(\mathrm{~m})\end{array}$ & $\begin{array}{c}\Delta_{u} \\
(\mathrm{~m}) \\
\end{array}$ & $\begin{array}{c}M_{y} \\
(k N-m)\end{array}$ & $\mu_{\Delta}$ & $\Delta_{y}^{*}$ & $\Delta_{\mathrm{u}}^{*}$ & $\mathbf{M}_{\mathrm{Y}}^{*}$ & $\boldsymbol{\mu}_{\Delta}$ & $\Delta_{y}^{*}$ & $\Delta_{\mathrm{u}}^{*}$ & $\mathbf{M}_{\mathrm{Y}}^{*}$ & $\boldsymbol{\mu}_{\Delta}$ \\
\hline 1 & 0,14 & 0,43 & 2777 & 3,0 & 1,0 & 2,8 & 0,7 & 8,2 & 1,0 & 2,7 & 0,7 & 8,0 \\
\hline 2 & 0,14 & 0,48 & 2563 & 3,3 & 1,1 & 2,5 & 0,7 & 7,5 & 1,1 & 2,5 & 0,7 & 7,3 \\
\hline 3 & 0,12 & 0,40 & 4408 & 3,2 & 1,1 & 2,8 & 0,7 & 8,6 & 1,1 & 3,0 & 0,8 & 8,6 \\
\hline 4 & 0,14 & 0,44 & 2543 & 3,2 & 1,3 & 2,8 & 0,6 & 6,8 & 1,2 & 2,6 & 0,6 & 7,1 \\
\hline 5 & 0,15 & 0,55 & 1648 & 3,8 & 1,1 & 2,0 & 0,9 & 6,6 & 1,2 & 2,1 & 0,9 & 6,8 \\
\hline 6 & 0,27 & 0,81 & 11640 & 3,0 & 1,1 & 2,9 & 0,6 & 8,2 & 1,1 & 3,0 & 0,6 & 8,2 \\
\hline 7 & 0,28 & 1,16 & 10910 & 4,2 & 1,1 & 2,0 & 0,6 & 7,8 & 1,1 & 2,0 & 0,6 & 7,6 \\
\hline 8 & 0,29 & 0,94 & 10830 & 3,3 & 0,9 & 1,9 & 0,7 & 7,0 & 1,1 & 2,5 & 0,7 & 7,5 \\
\hline 9 & 0,26 & 0,81 & 17200 & 3,1 & 1,0 & 2,7 & 0,8 & 8,1 & 1,1 & 2,9 & 0,8 & 8,1 \\
\hline 10 & 0,34 & 0,90 & 11220 & 2,7 & 1,0 & 2,5 & 0,7 & 6,7 & 1,0 & 2,6 & 0,7 & 6,8 \\
\hline 11 & 0,41 & 1,23 & 22270 & 3,0 & 1,1 & 2,8 & 0,8 & 7,7 & 1,1 & 3,0 & 0,8 & 8,0 \\
\hline 12 & 0,39 & 1,32 & 19620 & 3,4 & 1,2 & 2,6 & 0,8 & 7,4 & 1,2 & 2,6 & 0,8 & 7,3 \\
\hline 13 & 0,52 & 1,40 & 23950 & 2,7 & 1,0 & 2,5 & 0,7 & 7,1 & 1,0 & 2,5 & 0,7 & 7,0 \\
\hline 14 & 0,42 & 1,60 & 26880 & 3,8 & 1,1 & 1,9 & 0,7 & 6,6 & 1,1 & 1,9 & 0,7 & 6,6 \\
\hline 15 & 0,43 & 1,09 & 36160 & 2,5 & 0,9 & 2,8 & 0,9 & 7,5 & 1,1 & 3,3 & 0,9 & 7,6 \\
\hline
\end{tabular}

Fuente: elaboración propia.

expuesto por Moehle (2015). En relación a la resistencia nominal a flexión de los muros DMO y DES, se encontró que estas son, en promedio, 1,3 veces menor comparadas con la de los muros DMI.

\section{CONCLUSIONES Y TRABAJO FUTURO}

Los resultados obtenidos a partir del estudio analítico desarrollado permitieron establecer las siguientes conclusiones:

- El uso de un grado de capacidad de disipación de energía mayor al mínimo establecido por el reglamento NSR-10 para zonas de amenaza sísmica baja puede generar menores consumos de acero de refuerzo en muros estructurales de concreto reforzado fundidos en sitio. Esto obedece a que a pesar de que los requisitos de ductilidad para muros con capacidad moderada y especial requieren una mayor cantidad de acero transversal, como resultado de exigencias más estrictas en el confinamiento para los elementos de borde, las cuantías de acero por flexión y cortante en el alma del muro disminuyen considerablemente y controlan el valor total de acero requerido en el muro.

- Respecto al punto anterior, y para la zona de amenaza sísmica estudiada, la mayor reducción se logra cuando en el diseño se emplean muros con densidades de muro intermedias y altas, espesores mayores a 0,15 m, y capacidad de disipación moderada.

- El uso de capacidades de disipación de energía especial en el diseño de los muros puede resultar en mayores consumos de acero comparadas con las del diseño obtenido con capacidad mínima, sobre todo si el ingeniero proyectista emplea como criterio de diseño cuantías mínimas longitudinales y transversales iguales al 0,25\%, omitiendo la reducción permitida en el código NSR-10 en función de la demanda a cortante.

- Se confirmó que los muros con capacidad de disipación moderada y especial presentan una mayor capacidad de desplazamiento comparados con los muros de capacidad mínima, lo cual sigue el objetivo de diseño de retrasar el potencial colapso mediante un aumento en la ductilidad. 
- El hecho de que los muros con capacidad mínima presenten mayores valores de resistencia a flexión que los muros con capacidad moderada y especial genera el cuestionamiento de determinar el nivel de desempeño y el grado de daño que podrían tener los muros bajo la demanda sísmica real de la zona, o incluso bajo otras fuentes de demandas laterales como las acciones impuestas por el viento. Lo anterior puede ser explorado mediante una combinación de ensayos experimentales o análisis no lineales estáticos y dinámicos. Esto permitirá concluir si, en efecto, la decisión de usar un grado de capacidad de disipación de energía diferente al mínimo establecido para la zona no solo resulta en una reducción en los costos de la estructura, sino que también mejora la respuesta global sísmica de la edificación.

\section{FINANCIAMIENTO}

El estudio presentado en este artículo fue financiado por la Vicerrectoría de Investigación y Extensión de la Universidad Industrial de Santander, mediante el proyecto VIE-1372. Las conclusiones reflejan los puntos de vista de los autores y no aquellas del patrocinador.

\section{REFERENCIAS BIBLIOGRÁFICAS}

Abo-Shadi, N.; Saiidi, M. y Sanders, D. (2000). Seismic Response of Reinforced Concrete Bridge Pier Walls in the Weak Direction. Búfalo: Multidisciplinary Center for Earthquake Engineering Research.

Arteta, C., \& Moehle, J. (2015). Comportamiento Experimental de elementos de borde de muros con capacidad de disipación de energía especial (DES). VII Congreso Nacional de Ingeniería Sísmica. Bogotá.

ASCE (2013). ASCE/SEI Standard 41-13: Seismic Evaluation and Retrofit of Existing Buildings. American Society of Civil Engineers, Reston, Virginia, USA.

Asociación Colombiana de Ingeniería Sísmica (AIS) (2012). Reglamento colombiano de construcciones sismo-resistentes. Bogotá.
Blandón, C. y Bonett, R. (2013). Evaluación numérica y experimental del comportamiento de muros esbeltos de concreto reforzado. VI Congreso de Ingeniería Sísmica. Bucaramanga.

Blandón, C., Rave, J. y Bonett, R. (2015). Comportamiento de muros delgados de concreto reforzado ante cargas laterales. VII Congreso Sísmica. Bogotá.

Carrillo, J.; González, G. y Rubiano, A. (2014). Displacement ductility for seismic design of RC walls for low-rise housing. Latin American Journal of Solids and Structures, 11(4), 725-737.

Carrillo, J.; Sánchez-Cruz, M. y Viviescas, A. (2014). Strains on steel reinforcement of low-rise concrete walls during shake table tests. Ingeniería e Investigación, 34(1), 36-41.

Computers \& Structures Inc. (2009). CSI Analysis references Manual for SAP 2000, Etabs, Safe. CSI.

Hidalgo, P., Ledezma, C., \& Jordán, R. (2002). Seismic behavior of Squat Reinforced Concrete Shear Walls. Earthquake Spectra, 18(2), 187-208.

Mander, J.; Priestley, M. y Park, R. (1994). Theoretical Stress-Strain Model for Confined Concrete. Journal of Structural Engineering, 114(8), 1804-1826.

Moehle, J. (2015). Seismic Design of Reinforced Concrete Buildings. 1a. ed. Nueva York: McGraw-Hill Education.

Naranjo, C.; Marulanda, J. y Madera, S. (2015). Modelo analítico para la estimación de la respuesta histerética de muros delgados de concreto reforzado. VII Congreso Nacional de Ingeniería Sísmica. Bogotá.

Urrego, H. y Bonett, R. (2010). A Displacement based Design Method for Mediumrise Reinforced Concrete Walls. Dyna, 77(163), 1325.

Wallace, John W. (2007). Modelling Issues for Tall Reinforced Concrete Core Wall Buildings. The Structural Design off Tall and Special Buildings, 16 (5), 615-632.

Wood, S. (2005). Design of R/C Structural Walls: Balancing Toughness and Stiffness. En: P. Fajfar y $\mathrm{H}$. Krawinkler. Nonlinear Seismic Analyisis and Design of Reinforced Concrete Buildings (pp. 175-185). Nueva York: Elsevier Applied Science.

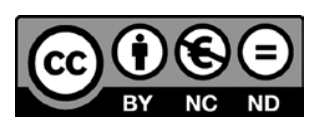

Tecnura • p-ISSN: 0123-921X • e-ISSN: 2248-7638 • Vol. 20 No. $50 \bullet$ Octubre - Diciembre $2016 \bullet$ pp. 15-28 